\title{
Pro-inflammatory/Th1 gene expression shift in high glucose stimulated mesangial cells and tubular epithelial cells
}

Yasunori Iwata1, 2, Kengo Furuichi2, 3, Shinichi Hashimoto4, 6, Kiyonobu Yokota4, Haruka Yasuda ${ }^{4}$, Norihiko Sakai2, 3, Shinji Kitajima ${ }^{2,5}$, Tadashi Toyama ${ }^{2,5}$, Yasuyuki Shinozaki2, 4, Akihiro Sagara $^{2,5}$, Kouji Matsushima ${ }^{6}$, Shuichi Kaneko ${ }^{5}$ and Takashi $\mathrm{Wada}^{2,4}$

${ }^{1}$ Division of Infection Control, 2Division of Nephrology, ${ }^{3}$ Division of Blood Purification, ${ }^{4}$ Department of Laboratory Medicine, ${ }^{5}$ Department of Disease Control and Homeostasis, Kanazawa University, ${ }^{6}$ Department of Molecular Preventive Medicine, University of Tokyo, Tokyo, Japan.

Key words; Diabetic nephropathy, inflammation, genome-wide analysis, mesangial cells, tubular epithelial cells

Short running title; Immune balance of renal resident cells in diabetic nephropathy.

Corresponding author and reprints request: Dr. Yasunori Iwata

Division of Nephrology

Division of Infection Control,

Kanazawa University

13-1 Takara-machi, Kanazawa 920-8641, Japan

tel +81-76-265-2000 (ext 2851)

fax $+81-76-234-4250$

e-mail: iwata-knz@umin.ac.jp 


\section{Abstract}

Diabetic nephropathy (DN) is a major cause of end stage kidney disease and a strong risk factor for cardiovascular diseases. Growing data show chronic inflammation plays an important role for the progression of DN. As for the immune cells, antiinflammatory leukocytes as well as pro-inflammatory leukocytes play an important role in DN. In addition to leukocytes, renal resident cells contribute to the inflammatory process of DN. However, precise functions, phenotypes and immune balance of renal resident cells remain to be explored. Therefore, we hypothesized that the aberrant immune balance of renal resident cells contributes to the pathogenesis of DN. To explore this possibility, we performed genome-wide transcriptome profiling in mesangial cells and tubular epithelial cells (TECs), which were stimulated by high glucose (HG) and detected the expression of inflammation associated genes. HG increased the mRNA expression of oxidative stress, inflammasome and mammalian target of rapamycin (mTOR) related genes in mesangial cells. Pro-inflammatory/Th1 gene expression was upregulated, but Th2 related gene expression was downregulated in mesangial cells. In TECs, HG stimulation increased pro-inflammatory/Th1/Th2 gene expression. Phosphorylation of signaling proteins shifted towards pro-inflammatory phenotype with suppressed phosphorylation of Th2 related signaling in TECs. The data taken together indicate that HG shifts the immune balance toward pro-inflammaotry/Th1 phenotype in mesangial cells and TECs, which might initiate and/or prolong inflammation, thereby resulting in diabetic nephropathy. 


\section{Introduction}

The number of patients with end stage kidney diseases (ESKD) is increasing worldwide. Especially, diabetic nephropathy (DN) is one of the major causes of ESKD in developed countries. Not only the high incidence of ESKD, but also the high mortality from cardiovascular diseases has been observed in patients with DN [1]. Therefore, identifying molecular mechanisms that mediate the progression of DN is an important issue to uncover novel therapeutic targets.

Growing evidence suggests that chronic inflammation plays key roles for the progression of $\mathrm{DN}[2-4]$. Recent studies revealed that macrophages $(\mathrm{M} \phi)$ and $\mathrm{T}$ cells contribute to the pathogenesis of $\mathrm{DN}$ in both rodent and human models. Activated $\mathrm{M} \phi$ and T cells induce and aggravate the kidney injury, resulting in chronic kidney failure. Recently, $\mathrm{M} \phi$ are categorized as inflammatory (M1) and anti-inflammatory (M2) phenotype according to the cytokine profile and cell surface marker [5]. M2 M $\phi$ as well as $\mathrm{M} 1 \mathrm{M} \phi$ has been reported to contribute to the pathogenesis of $\mathrm{DN}$ [6-8]. CD4 ${ }^{+} \mathrm{T}$ cell populations are also broadly and simplistically divided into 4 types, Th1, Th2, Th17 and regulatory T cells based on the function [9]. Each phenotype of Th cell has been reported as a key player for the progression of $\mathrm{DN}$ [10-12]. These data suggest that the immune balance of inflammatory cells affect the prognosis of $\mathrm{DN}$.

Not only accumulated leukocytes, but also renal resident cells orchestrate in the inflammatory processes in DN. Activated renal resident cells secret various cytokines/chemokines $[13,14]$. However, precise functions, phenotypes and immune balance of renal resident cells remain to be explored in DN. Therefore, we hypothesized that the aberrant immune balance of renal resident cells may contribute to the pathogenesis of DM. To explore this hypothesis, we performed genome-wide 
transcriptome profiling in mesangial cells and tubular epithelial cells (TECs), which were stimulated by high glucose (HG), and identified the expression of inflammatory associated genes.

\section{Materials and methods}

Cell culture

Normal human mesangial cells (Lonza, Basel, Switzerland) were cultured in mesangial culture kit (Lonza). Human immortalized TECs, HK-2 (ATCC, Manassas, VA) was cultured in 10\% FBS DMEM (Life technologies, Tokyo) with $100 \mu \mathrm{g} / \mathrm{ml}$ streptomycin and $100 \mathrm{U} / \mathrm{ml}$ penicillin. All the cells were grown in a humidified atmosphere (5\% CO2/95\% air) at $37^{\circ} \mathrm{C}$ and were seeded onto six-well cell culture cluster (Corning Incorporated, Corning, NY). The cells were stimulated with $30 \mathrm{mM}$ glucose (Wako, Tokyo) for 24 hours for hyperglycemic stimulation. For osmotic control, the cells were cultured with $30 \mathrm{mM}$ mannitol (Wako) for 24 hours.

\section{SAGE analysis using Ion Torrent PGM ${ }^{T M}$.}

Gene expression analysis was performed by SAGE method and NGS. Briefly, RNA was isolated using mRNA Isolation kit (Sigma-Aldolich, St louis, MO). NGS data from the PGMTM was generated from $1 \mu \mathrm{g}$ of total RNA isolated from cell line. SAGE libraries were constructed using the SOLiD SAGETM kit from Life Technologies (Life Technologies) according to manufacturer's protocol. DNA was recovered from the agarose gel using PureLink Gel Extraction kit (Life Technologies). DNA fragments of SAGE construct were analysed on the Agilent Bioanalyzer using the High Sensitivity Kit (Agilent, Santa Clara, CA). Template preparation and emulsion PCR, and Ion 
Sphere Particles (ISP) enrichment was done using the Ion Xpress Template kit (Life Technologies) according to the manufacturer's instructions. The quality of the resultant ISPs was assessed using Qubit 2.0 Fluorometer (Life Technologies), and were loaded and sequenced on a 318 chip (Life Technologies). The complete data sets from these experiments have been deposited in the NCBI Gene Expression Omnibus (http://www.ncbi.nlm.nih.gov/geo; accession number GSE52734).

\section{Mapping from NGS data}

For each of the samples, the PGM ${ }^{\mathrm{TM}}$ raw reads were aligned against the human refseq genes (UCSC, http/hgdownload.cse.ucsc.edu/) using BWA 0.6.2 which uses the 25_1 mapping parameter. We generated unique gene counts by excluding reads that mapped to contigs of more than one gene. Reads mapping to several contigs within an isogroup were only counted once.

\section{Antibodies}

The following antibodies were used for flow cytometric analysis; Phycoerythrin (PE) conjugated anti-phosphorylated p38 (612565; BD Biosciences, San Jose, CA), PE-conjugated anti-phosphorylated STAT1 (562674; BD Biosciences), PE-conjugated anti-phosphorylated STAT3 (558557; $\quad$ BD $\quad$ Biosciences), PE-conjugated anti-phosphorylated STAT5 (61257; BD Biosciences), PE-conjugated anti-phosphorylated STAT6 (612701; BD Biosciences).

\section{Flow cytometric analysis}

To detect phosphorylated intracellular signaling, cells were fixed and permeabilized 
using BD Phosflow ${ }^{\mathrm{TM}}$ in accordance with the manufacturer's protocol (BD Biosciences). Then, the cells were stained with antibody. We collected 10000 cultured cells using FACSCalibur (BD Biosciences) and analyzed data using FlowJo software 9.3 (Tree Star, Palo Alto, CA).

\section{Reconstitution of signal transduction pathway}

To identify the signal transduction pathway, each gene was reconstituted according to Kyoto Encyclopedia of Genes and Genomes (KEGG: http://www.genome.jp/kegg/) database.

\section{Statistics}

Statistical analysis was performed as described before [15]. Data represent the expression ratio (HG stimulated /osmotic control).

\section{Result}

High glucose $(H G)$ increased the $m R N A$ expression of oxidative stress, inflammasome and mammalian target of rapamycin (mTOR) related genes.

Oxidative stress $[16,17]$ has been regarded as intracellular mediator in HG induced inflammation. Thus, we examined the mRNA expression of oxidative stress associated genes. Among them, reactive oxygen species modulater 1 (ROMO) and protein kinase $\mathrm{C}$ (PKC) alpha (PRKCA) mRNA were increased in HG stimulated mesangial cells (Fig.1). Recent study revealed that PKC signaling is closely related to mTOR signaling $[18,19]$. Therefore, we analyzed mTOR signaling related genes. Interestingly, the relative gene expression of mTOR and its downstream signaling, translation initiation factor $4 \mathrm{~B}$ 
(EIF4B) were upregulated. Moreover, thioredoxin interacting protein (TXNIP) mRNA, which has the potential to activate the NOD-like receptor family, pyrin domain containing (NLRP) 3 inflammasome [20], was also increased in HG stimulated mesangial cell. In addition, transcription factor, activated protein (AP)-1 (JUN, FOSL2), C-C chemokine ligand (CCL) 2 and tumor necrosis factor (TNF)- $\alpha$ mRNA were also increased higher in HG stimulated mesangial cells as compared to osmotic control.

HG stimulated mesangial cells were skewed toward an inflammatory phenotype.

As HG increased the mRNA expression levels of inflammatory signal mediators, we look into whether HG stimulation shifted the cell phenotype toward an inflammatory population. Inflammatory cytokines/chemokines and their receptors, such as CCL2, TNF- $\alpha$ and interleukin(IL)-1 receptor mRNA expression were increased by the HG stimulation in mesangial cells. Moreover, mitogen activated protein kinase (MAPK) mRNA expression was also increased in HG stimulated mesangial cells. In contrast, Th2 related cytokine receptors and intracellular signaling molecules showed the reduced mRNA expression levels (Table.1). Taken together, HG stimulation increased mRNA expression of the pro-inflammatory molecules and decreased Th2 related molecules in mesangial cells.

Increased mRNA expression of inflammatory cytokines/chemokines and Th2 type cytokine receptors in HG stimulated TECS.

Next, we examined the functional phenotype of HG stimulated TECs. Stimulated TECs showed the increased mRMA levels of inflammatory cytokines/chemokines and their receptors, such as TNF- $\alpha$, IL-1 receptor, IL-18, IL-12 and IL-6. In addition, mRNA 
expression of Th2 related cytokine receptors, IL-10 receptor and IL-13 receptor, also increased via HG stimulation (Table. 2).

Phosphorylation of intracellular signaling molecules were increased in HG stimulated mesangial cells and TECS.

Intracellular signaling has crucial roles in cytokines/chemokines- mediated cellular reactions, such as proliferation, differentiation and activation. The phosphorylation of signaling proteins is essential for the activation of the signal transduction pathways. Therefore, we examined the phosphorylation of signaling proteins in HG stimulated mesangial cells and TECs. Phosphorylated form of p38 MAPK and signal transducer and activator of transcription (STAT) 3, which are related to pro-inflammatory/Th1 signaling [21] were significantly higher in 24 hours HG stimulated mesangial cells and TECs. Conversely, phosphorylated forms of STAT5 and STAT6, which are associated with Th2 type signaling transductions[21], were significantly lower in 24 hours HG stimulated TECs (Fig.2). These data suggest the shift of the immune balance toward pro-inflammtory phenotype in mesangial cells and TECs stimulated with HG.

\section{Discussion}

We now report that HG stimulation increases pro-inflammatory/Th1 gene expression, but decreases Th2 related gene expression in mesangial cells. In TECs, HG stimulation increased pro-inflammatory/Th1/Th2 gene expression. Phosphorylation of signaling proteins shifted towards pro-inflammatory phenotype with suppressed phosphorylation of Th2 related signaling proteins in mesangial cells and TECs. The data taken together indicate that HG shifts the immune balance toward pro-inflammaotry/Th1 phenotype in 
mesangial cells and TECs, which might initiate and/or prolong inflammation, thereby resulting in diabetic nephropathy.

Growing evidence suggests that chronic inflammation plays important roles in the progression of DN [2-4]. Not only the leukocytes, but also renal resident cells, including mesangial cells and TECs participate in the pathogenesis of DN. HG stimulation induced the gene expression of PKC and ROS related proteins in mesangial cells. PKC and ROS related proteins have been reported as the key signaling for HG induced mesangial cell damage $[16,17]$. Therefore, our data suggest HG stimulation activated intracellular signaling pathway in mesangial cells. Interestingly, the relative gene expression of mTOR and its downstream signaling, translation initiation factor $4 \mathrm{~B}$ (EIF4B) were upregulated. Supporting this notion, the recent study revealed that PKC signaling is closely related to mTOR signaling [18, 19]. Moreover, mTOR expression has been reported in mesangial cells and podocytes in $\mathrm{DN}[22,23]$. In addition, thioredoxin interacting protein (TXNIP) gene expression was also increased in HG stimulated mesangial cells. Lerner et al. reported that increased TXNIP activate NLRP 3 inflammasome, causing procaspase-1 cleavage and IL-1B secretion [20]. Another group also reported the involvement of inflammasome in DN [24]. Thus, our data suggest HG stimulation activate mTOR signaling and inflammasome in cultured mesangial cells. However, more precise analysis are needed to confirm if these signaling pathway is really involved in $\mathrm{DN}$.

Recently, $\mathrm{M} \phi$ are categorized as inflammatory (M1) and anti-inflammatory (M2) phenotype according to the cytokine profile and cell surface markers [5]. CD4 $4^{+} \mathrm{T}$ cell populations are also divided into 4 types, Th1, Th2, Th17 and regulatory T cells based on the function [9]. Orchestration of inflammation by pro-inflammatory cells with 
anti-inflammatory cells has an impact on the process of progressive kidney diseases including DN. In this study, mesangial cells and TECs displayed pro-inflammatory phenotype with the reduction of Th2 related genes via HG stimulation. In support of our findings, Min et al. reported that HG increases TNF- $\alpha$ and IL-6 secretion in mesangial cells [25]. Also tubular epithelial cells have been reported as an important source for cytokines/cemokines in diabetes (14). Moreover, suppression of JAK/STAT signaling decreased the expression of pro-inflammatory cytokines/chemokines, resulting in improved kidney injury in a rat diabetes model [26]. In addition, we have reported that repairing TECs from hypoxia injury releases mediator that skews M1 M $\phi$ toward M2 phenotype [27]. The data taken together indicate that renal resident cells such as mesangial cells and TECs orchestrate in the inflammatory processes with changing the immune balance.

As for the intracellular signaling pathway, phosphorylation plays critical roles in signal transduction. Thus, we analyzed the phosphorylation of each molecule. HG stimulation for 24 hours increased the phosphorylation of p38 and STAT3. P38 MAPK is a key signaling for inflammatory cytokines/chemokines, thereby contributing to the progression of inflammatory kidney diseases [28-31], including DN [8]. STAT3 pathway is required for IL-23/IL-17 signaling, thereby leading helper T cell to Th17 axis [21]. Supporting our notion, STAT3 expression has been reported in mesangial cell [32], mediating cell proliferation and activation. Moreover, Ranganathan et al. reported the increased collagen expression via STAT3 activation in TECs [33]. However, the association STAT3 and Th17 axis is not clear in renal resident cells. Loverre et al reported the IL-17 expression in TECs in renal transplant recipients with acute rejection, though STAT3 activation was not mentioned [34]. 
In contrast, STAT5 and STAT6 phosphorylation were decreased in HG stimulated TEC. IL-2/STAT5 and IL-4/STAT6 signalings promote Th2 polarization in CD4 helper T cell $[21,35]$. Interestingly, STAT6 signaling showed reno-protective potential in crescentic glomerulonephritis [36] and ischemia-reperfusion (I/R) injury [37]. Moreover, STAT5 signaling plays a central role in erythropoietin mediated tissue protection in I/R injury [38] and cisplatin induced acute kidney injury [39]. Therefore, the reduction of STAT6 and STAT5 signaling might suggest the blunted protective effect in HG stimulated TECs. However, the precise role of STAT5 and STAT6 signalings in DN remains to be investigated.

In conclusion, HG stimulation changes the immune balance toward inflammatory phenotype in mesangial cells and TECs, which might initiate and/or prolong inflammation, thereby resulting in DN. We anticipate that future studies elucidate the precise mechanisms of aberrant immune balance and thereby provide novel therapeutic approaches to DN.

\section{Acknowledgement}

This study was supported by grants from the Ministry of Education, Science, Sports, and Culture, Grant-in-Aids for Diabetic Nephropathy Research and for Diabetic Nephropathy and Nephrosclerosis Research, Core Research for Evolutional Science and Technology (CREST) of the Japan Science, and Technology Corporation (JST) from ministry of Health, labor and Welfare of Japan 


\section{References}

[1] Afkarian M, Sachs MC, Kestenbaum B, et al. Kidney disease and increased mortality risk in type 2 diabetes. J Am Soc Nephrol. 24 (2013) 302-8.

[2] Hara A, Sakai N, Furuichi K, et al. CCL2/CCR2 augments the production of transforming growth factor-beta1, type 1 collagen and CCL2 by human CD45-/collagen 1-positive cells under high glucose concentrations. Clin Exp Nephrol. 6 (2013) [Epub ahead of print]

[3] Wada J, Makino H. Inflammation and the pathogenesis of diabetic nephropathy. Clin Sci (Lond).124 (2013) 139-52.

[4] Lim AK, Tesch GH. Inflammation_in_diabetic nephropathy. Mediators Inflamm. (2012) 146154

[5] Sica A, Mantovani A. Macrophage_plasticity and polarization: in vivo veritas. J Clin Invest. 122 (2012) 787-95.

[6] Zheng D, Wang Y, Cao Q, et al. Transfused macrophages ameliorate pancreatic and renal injury in murine diabetes mellitus. Nephron Exp Nephrol. 118 (2011) e87-99.

[7] Cao Q, Wang C, Zheng D, et al. IL-25 induces M2 macrophages and reduces renal_injury_in proteinuric_kidneydisease. J Am Soc Nephrol. 22 (2011) 1229-39.

[8] Sakai N, Wada T, Furuichi K, et al. Involvement of extracellular signal-regulated kinase and p38 in human_diabetic nephropathy. Am J Kidney Dis. 45 (2005) 54-65.

[9] Vahedi G, C Poholek A, Hand TW, et al. Helper T-cell identity and evolution of differential transcriptomes and epigenomes. Immunol Rev. 252 (2013) 24-40.

[10] Moon JY, Jeong KH, Lee TW, et al. Aberrant recruitment and activation of T cells in diabetic nephropathy. Am J Nephrol. 35 (2012) 164-74.

[11] Eller K, Kirsch A, Wolf AM, et al. Potential role of regulatory T cells in reversing 
obesity-linked insulin resistance and diabetic nephropathy. Diabetes 60 (2011) 2954-62.

[12] Wu CC, Sytwu HK, Lu KC, et al. Role of T cells in type 2 diabetic nephropathy. Exp Diabetes Res. (2011) 514738.

[13] Scindia YM, Deshmukh US, Bagavant H. Mesangial pathology in glomerular disease: targets for therapeutic intervention. Adv Drug Deliv Rev. 62 (2010) 1337-43.

[14] Tang SC, Lai KN. The pathogenic role of the renal proximal tubular cell in diabetic nephropathy. Nephrol Dial Transplant. 27 (2012) 3049-56.

[15] Kal AJ, van Zonneveld AJ, Benes V, et al. Dynamics of gene expression revealed by comparison of serial analysis of gene expression transcript profiles from yeast grown on two different carbon sources. Mol Biol Cell. 10 (1999) 1859-72.

[16] Zhang Y, Peng F, Gao B, et al. High glucose-induced RhoA activation requires caveolae and_PKC61-mediated ROS generation. Am J Physiol Renal Physiol. 302(2012) F159-72.

[17] Thallas-Bonke V, Thorpe SR, Coughlan MT, et al. Inhibition of NADPH oxidase prevents advanced glycation end product-mediateddamage in diabetic nephropathy through a protein kinase C-alpha-dependent pathway. Diabetes. 57 (2008) 460-9.

[18] Velazquez-Garcia S, Valle S, Rosa TC, et al. Activation of protein kinase C- $\zeta$ in pancreatic B-cells in vivo improves glucose tolerance and induces B-cell expansion via mTOR activation. Diabetes.60(2011) 2546-59.

[19] Lee K, Gudapati P, Dragovic S, et al. Mammalian target of rapamycin protein complex 2 regulates differentiation of Th1 and Th2 cell subsets via distinct 
signaling pathways. Immunity. 32 (2010) 743-53.

[20] Lerner AG, Upton JP, Praveen PV, et al. IRE1a induces thioredoxin-interacting protein to activate the NLRP3 inflammasome and promote programmed cell death under irremediable ER stress. Cell Metab. 16 (2012) 250-64.

[21] O'Shea JJ, Paul WE. Mechanisms underlying lineage commitment and plasticity of helper CD4+ T cells. Science. 327 (2010) 1098-102.

[22] Inoki K, Mori H, Wang J, et al. mTORC1 activation in podocytes is a critical step in the development of diabetic nephropathy in mice. J Clin Invest. 121 (2011) 2181-96.

[23] Buller CL, Heilig CW, Brosius FC 3rd. GLUT1 enhances mTOR activity independently of TSC2 and AMPK. Am J Physiol Renal Physiol. 301 (2011) F588-96.

[24] Chen K, Zhang J, Zhang W, et al. ATP-P2X4 signaling mediates NLRP3 inflammasome activation: A novel pathway of diabetic nephropathy. Int J Biochem Cell Biol. 45 (2013) 932-43.

[25] Min D, Lyons JG, Bonner J, et al. Mesangial cell-derived factors alter monocyte activation and function through inflammatory pathways: possible pathogenic role in diabetic nephropathy. Am J Physiol Renal Physiol. 297 (2009) F1229-37.

[26] Ortiz-Muñoz G, Lopez-Parra V, Lopez-Franco O, et al. Suppressors of cytokine signaling abrogate diabetic nephropathy. J Am Soc Nephrol. 21 (2010) 763-72.

[27] Iwata Y, Boström EA, Menke J, et al. Aberrant macrophages mediate defective kidney repair that triggers nephritis in lupus-susceptible mice. J Immunol. 188 (2012) 4568-80.

[28] Iwata Y, Wada T, Furuichi K, et al. p38 mitogen-activated protein kinase contributes to autoimmune renal injury in MRL-Faslpr mice. J Am Soc Nephrol. 14 
(2003) 57-67.

[29] Furuichi K, Wada T, Iwata Y, et al. Administration of FR167653, a new anti-inflammatory compound, prevents renal ischaemia/reperfusion injury in mice. Nephrol Dial Transplant. 17 (2002) 399-407.

[30] Wada T, Azuma H, Furuichi K, et al. Reduction in chronic allograft nephropathy by inhibition of p38 mitogen-activated protein kinase. Am J Nephrol. 26 (2006) 319-25.

[31] Iwata Y, Furuichi K, Sakai N, et al. Dendritic cells contribute to autoimmune kidney injury in MRL-Faslpr mice. J Rheumatol. 36 (2009) 306-14.

[32] Hirai T, Masaki T, Kuratsune M, et al. PDGF receptor tyrosine kinase inhibitor suppresses mesangial cell proliferation involving STAT3 activation. Clin Exp Immunol. 144 (2006) 353-61.

[33] Ranganathan P, Jayakumar C, Ramesh G. Proximal tubule-specific overexpression of netrin-1 suppresses acute kidney injury-induced interstitial fibrosis and glomerulosclerosis through suppression of IL-6/STAT3signaling. Am J Physiol Renal Physiol. 304 (2013) F1054-65.

[34] Loverre A, Tataranni T, Castellano G, et al. IL-17 expression by tubular epithelial cells in renal transplant recipients with acute antibody-mediated rejection. Am J Transplant. 11 (2011) 1248-59.

[35] Maier E, Duschl A, Horejs-Hoeck J. STAT6-dependent and -independent mechanisms in Th2 polarization. Eur J Immunol. 42 (2012) 2827-33.

[36] Summers SA, Phoon RK, Odobasic D, et al. Signal transducer and activation of transcription 6 (STAT6) regulates T helper type 1 (Th1) and Th17 nephritogenic immunity in experimental crescentic glomerulonephritis. Clin Exp Immunol. 166 (2011) 227-34. 
[37] Yokota N, Burne-Taney M, Racusen L, et al. Contrasting roles for STAT4 and STAT6 signal transduction pathways in murine renal ischemia-reperfusion injury. Am J Physiol Renal Physiol. 285 (2003) F319-25.

[38] Breggia AC, Wojchowski DM, Himmelfarb J JAK2/Y343/STAT5 signaling axis is required for erythropoietin-mediated protection against ischemic injury in primary renal tubular epithelial cells. Am J Physiol Renal Physiol. 295 (2008) F1689-95.

[39] Salahudeen AK, Haider N, Jenkins J, et al. Antiapoptotic properties of erythropoiesis-stimulating proteins in models of cisplatin-induced acute kidney injury. Am J Physiol Renal Physiol. 294 (2008) F1354-65. 


\section{Figure legend}

Figure 1

High glucose (HG) increased the mRNA expression of oxidative stress, inflammasome and mammalian target of rapamycin (mTOR) related genes. HG increased the mRNA expression of reactive oxygen species modulater 1 (ROMO), protein kinase $\mathrm{C}$ alpha (PRKCA) and thioredoxin interacting protein (TXNIP). Moreover, gene expression of mTOR and its downstream signaling, translation initiation factor 4B (EIF4B) were also upregulated. Data represents the ratio of gene expression (HG stimulated mesangial cells /mannitol treated mesangial cells).

Figure 2

Pro-inflammatory/Th17 related phosphorylated intracellular signaling were increased in HG stimulated mesangial cells and TECs. Phosphorylated p38 MAPK and STAT3 was significantly higher in 24 hours HG stimulated mesangial cells and TECs. Conversely, phosphorylated STAT5 and STAT6 were significantly lower in 24 hours HG stimulated TECs. Data represents means \pm SEM. ${ }^{*} \mathrm{p}<0.05,{ }^{* *} \mathrm{p}<0.01,{ }^{* * *} \mathrm{p}<0.001$ 


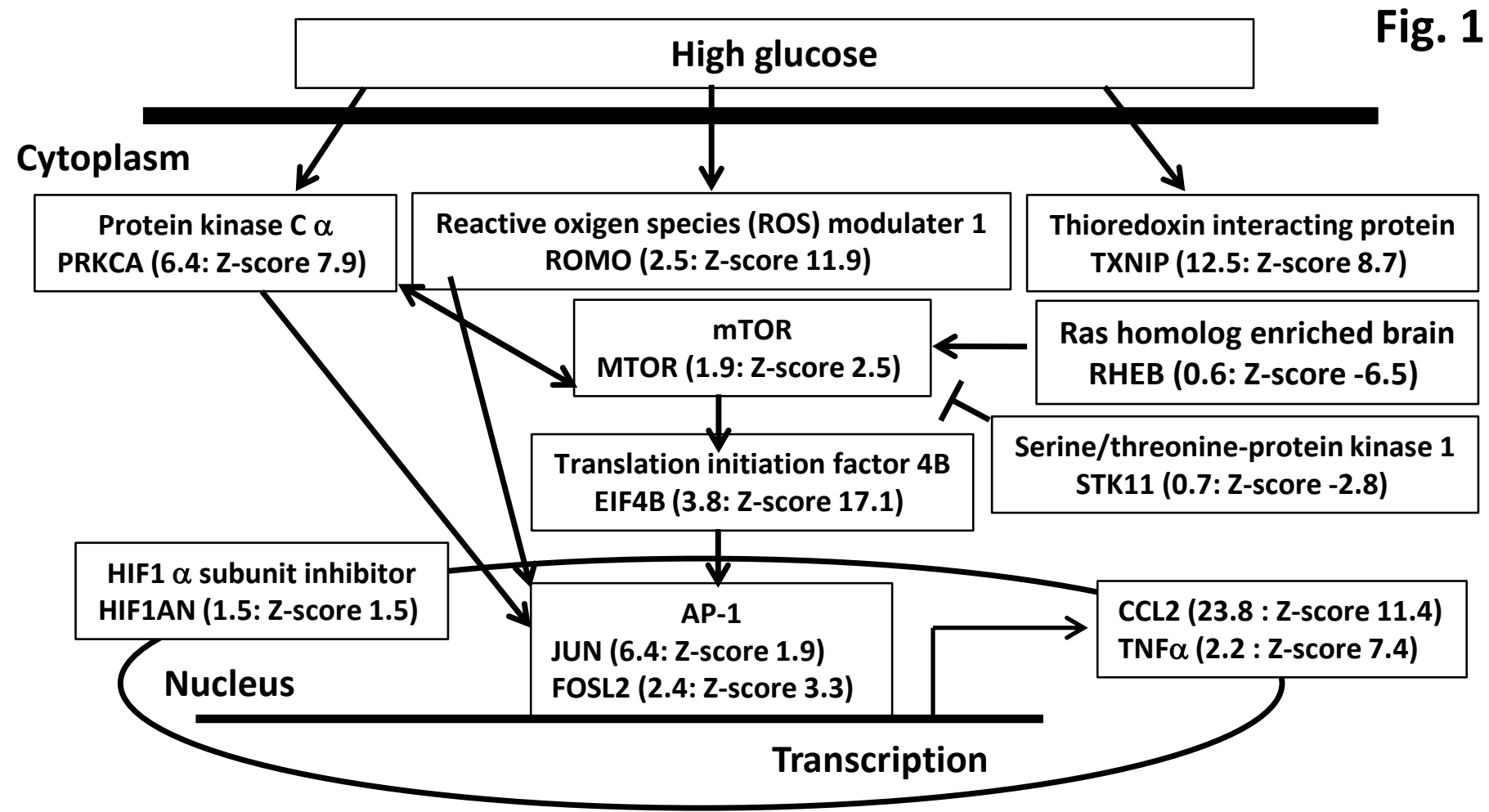



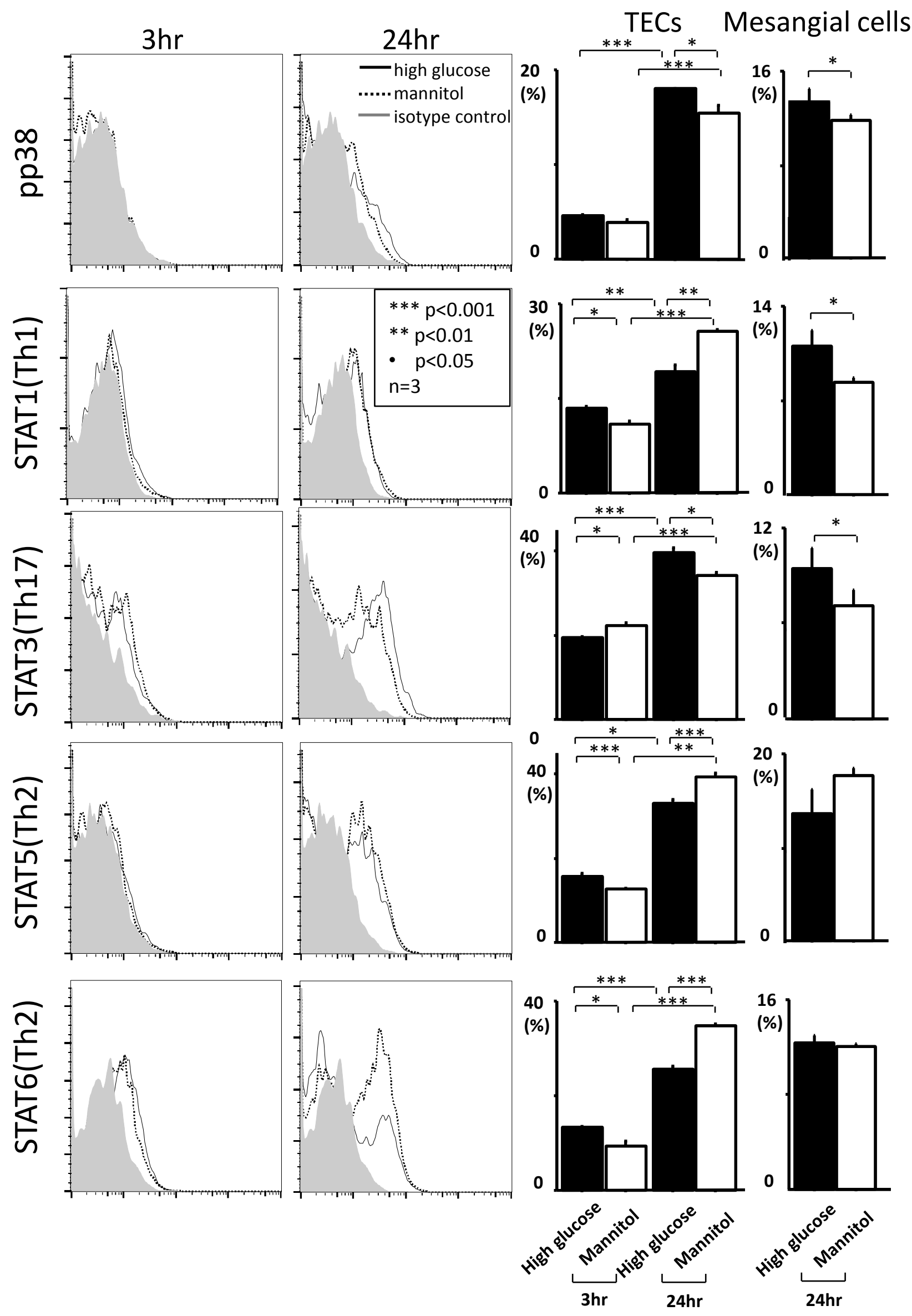


\begin{tabular}{|c|c|c|c|c|}
\hline \multirow[b]{2}{*}{ Pro-inflammatory } & \multicolumn{2}{|c|}{$\begin{array}{c}\text { Increased genes } \\
\text { (High glucose/Mannitol control) }\end{array}$} & \multicolumn{2}{|c|}{$\begin{array}{c}\text { Decreased genes } \\
\text { (High glucose/Mannitol control) }\end{array}$} \\
\hline & $\begin{array}{l}\text { CCL2 (23.8) } \\
\text { TNF } \alpha(2.2) \\
\text { IL-1R (2.0) }\end{array}$ & $\begin{array}{l}\text { MAPK1 (1.6) } \\
\text { MAPK3 (1.1) } \\
\text { MAPK6 (1.7) }\end{array}$ & & MAPK7 (0.7) \\
\hline Th1 & & STAT1 (1.3) & IFN- $\gamma \mathrm{R}(0.5)$ & \\
\hline Th2 & & & $\begin{array}{l}\text { IL-4R (0.5) } \\
\text { IL-10R } \beta(0.9) \\
\text { IL-13R } \beta(0.8)\end{array}$ & $\begin{array}{l}\text { STAT5 }(0.5) \\
\text { STAT6 }(0.5)\end{array}$ \\
\hline Th17 & IL-6ST (1.3) & & IL-6 (0.5) & STAT3 (0.3) \\
\hline TGF & & $\begin{array}{l}\text { SMAD1 (1.3) } \\
\text { SMAD2 (1.7) }\end{array}$ & & SMAD3 (0.4) \\
\hline
\end{tabular}

Table 1.

HG stimulated mesangial cells were skewed toward an inflammatory phenotype. Abbreviations are CCL: CC chemokine ligand, TNF: tumor necrosis factor, MAPK: mitogen activated protein kinase, STAT: signal transducer and activator of transcription, IFN: Interferon, IL: interleukin, SMAD: Sma and Mad related family. 


\begin{tabular}{c|l|l} 
& \multicolumn{1}{|c|}{$\begin{array}{c}\text { Increased genes } \\
\text { (High glucose/Mannitol control) }\end{array}$} & $\begin{array}{c}\text { Decreased genes } \\
\text { (High glucose/Mannitol control) }\end{array}$ \\
\hline Pro-inflammatory & $\begin{array}{l}\text { TNF } \alpha(1.5) \quad \text { MAPK1 (1.3) } \\
\text { IL-1R (3.0) }\end{array}$ & MAPK6 (0.6) \\
\hline Th1 & $\begin{array}{l}\text { IL-18 (1.8) STAT1 (2.8) } \\
\text { IL-12 (2.0) }\end{array}$ & IFN- $\gamma R$ (0.8) \\
\hline Th2 & $\begin{array}{l}\text { IL-10R } \beta(1.7) \\
\text { IL-13R } \alpha 1(2.0)\end{array}$ & \\
\hline Th17 & IL-6 (3.6) & TGF- $\beta 1(0.8)$
\end{tabular}

Table 2.

Increased mRNA expression of inflammatory cytokine/chemokine and Th2 type cytokine receptor in HG stimulated TECs.

Abbreviations are TNF: tumor necrosis factor, MAPK: mitogen activated protein kinase, STAT: signal transducer and activator of transcription, IFN: Interferon , IL: interleukin, TGF: transforming growth factor. 